\title{
Cells with Broken Left-Right Symmetry: Roles of Intrinsic Cell Chirality in Left-Right Asymmetric Epithelial Morphogenesis
}

\author{
Sosuke Utsunomiya, So Sakamura, Takeshi Sasamura, Tomoki Ishibashi ${ }^{\circledR}$, Chinami Maeda, \\ Mikiko Inaki * and Kenji Matsuno *(D) \\ Department of Biological Sciences, Graduate School of Science, Osaka University, Osaka 560-0043, Japan; \\ uts.199.ubosou@gmail.com (S.U.); u1tgah0s0@gmail.com (S.S.); sasamura@bio.sci.osaka-u.ac.jp (T.S.); \\ ishibaki@bio.sci.osaka-u.ac.jp (T.I.); mankind670@gmail.com (C.M.) \\ * Correspondence: inaki@bio.sci.osaka-u.ac.jp (M.I.); kmatsuno@bio.sci.osaka-u.ac.jp (K.M.)
}

Received: 22 December 2018; Accepted: 26 March 2019; Published: 8 April 2019

\begin{abstract}
Chirality is a fundamental feature in biology, from the molecular to the organismal level. An animal has chirality in the left-right asymmetric structure and function of its body. In general, chirality occurring at the molecular and organ/organism scales has been studied separately. However, recently, chirality was found at the cellular level in various species. This "cell chirality" can serve as a link between molecular chirality and that of an organ or animal. Cell chirality is observed in the structure, motility, and cytoplasmic dynamics of cells and the mechanisms of cell chirality formation are beginning to be understood. In all cases studied so far, proteins that interact chirally with F-actin, such as formin and myosin I, play essential roles in cell chirality formation or the switching of a cell's enantiomorphic state. Thus, the chirality of F-actin may represent the ultimate origin of cell chirality. Links between cell chirality and left-right body asymmetry are also starting to be revealed in various animal species. In this review, the mechanisms of cell chirality formation and its roles in left-right asymmetric development are discussed, with a focus on the fruit fly Drosophila, in which many of the pioneering studies were conducted.
\end{abstract}

Keywords: cell chirality; left-right asymmetry; myosin I; epithelial cell; gut; Drosophila

\section{Chirality Appears as a Hierarchical Structure in Biology}

Chirality has been a fascinating problem in biology. A substance is chiral if it cannot be superimposed on its mirror image. The chirality of biomolecules is fundamental for almost every event occurring in an organism. The specific three-dimensional structure of biopolymers, most of which are chiral, is an essential precondition underlying, for example, enzymatic reactions and molecular recognition. The chirality of biopolymers, including proteins and polysaccharides, depends on the homochirality of their building blocks, such as amino acids and sugars. A substance is homochiral if all of its constituent units have the same chiral form, designated as an enantiomer. In cells, amino acids are L-chiral, while sugars are D-chiral, except for some rare cases [1]. The origin of such homochirality is a fundamental question in understanding how life began. However, it is still not clear how and when homochirality first emerged [2]. 
Another fascinating chirality-related question in biology involves the directional left-right (LR) asymmetry of organ and body structures. Although links between molecular chirality and organ chirality have been proposed, these phenomena have mostly been studied independently of each other. In the middle of the 19th century, inversion of the LR asymmetric internal organs was reported in humans, although experimental approaches for understanding this observation at the time were severely limited [3]. In the early 20th century, Spemann and Huxley studied conjoined twins of newts, which were generated experimentally by tying embryos with a hair to only partially divide them in half. The laterality was always normal in the left-hand twin, but half of the right-hand twins showed LR inversion in their internal organs [4]. This was probably the first evidence that mechanisms directing LR polarity exist in developing embryos.

At about the same time, the genetic mechanisms controlling LR asymmetry attracted the attention of prominent geneticists. In Pulmonata (freshwater snails), mutations that affect the handedness of the shell coiling and the internal organs were isolated from natural populations [5]. One of the first mutations to be discovered was the inversed shell-coiling variant in the Lymnaea snail. Alfred Henry Sturtevant, a former student of Thomas Hunt Morgan, revealed that this mutant was recessive and maternally inherited [6]. Until recently, studies on biological chirality at the level of molecules and organisms were generally independent, and chirality appeared as a hierarchical structure in biology. Today, links between chirality at the molecular and organismal levels are slowly beginning to be understood [7-10]. Notably, between these two layers of biological chirality, chirality at the cellular level was discovered [10-17].

\section{Cells Have Chirality}

Once techniques for culturing various mammalian cells were established, potential chirality in the structures and motions of these cells was investigated in vitro [18]. The organization of the actin cytoskeleton was found to exhibit some chirality [19]. However, in general, the resolution of the analyses was not sufficient to confirm the chirality of cells until recently. Thus, chirality at the cellular level was not studied until modern techniques such as confocal microscopy and live imaging enabled the structures and motion of cells to be clearly detected in detail. These studies revealed that different cultured vertebrate cells had intrinsic chirality [20-25]. Chirality was observed in various cell characteristics, including their migration direction and trajectory, arrangement among other cells, and intracellular dynamics [20-25]. Similar techniques were also used in vivo to detect cell chirality in Drosophila [11]. In addition, Dictyostelium discoideum, a cellular slime mold, shows chirality in its locomotion at the single-cell stage [26]. These findings together show that chirality is a general characteristic of eukaryotic cells. These chiral behaviors of eukaryotic cells are generally designated as "cell chirality" and have been described in several reviews $[15,16,27,28]$. In other reviews in this special issue, the cell chirality of various cultured cells is discussed. In addition, the cell chirality observed in Pulmonata snails and Dictyostelium discoideum is described in other reviews in this special issue. Here, we will focus on cell chirality in the embryonic gut of Drosophila melanogaster, which was the first case of cell chirality identified in vivo (Figure 1). 

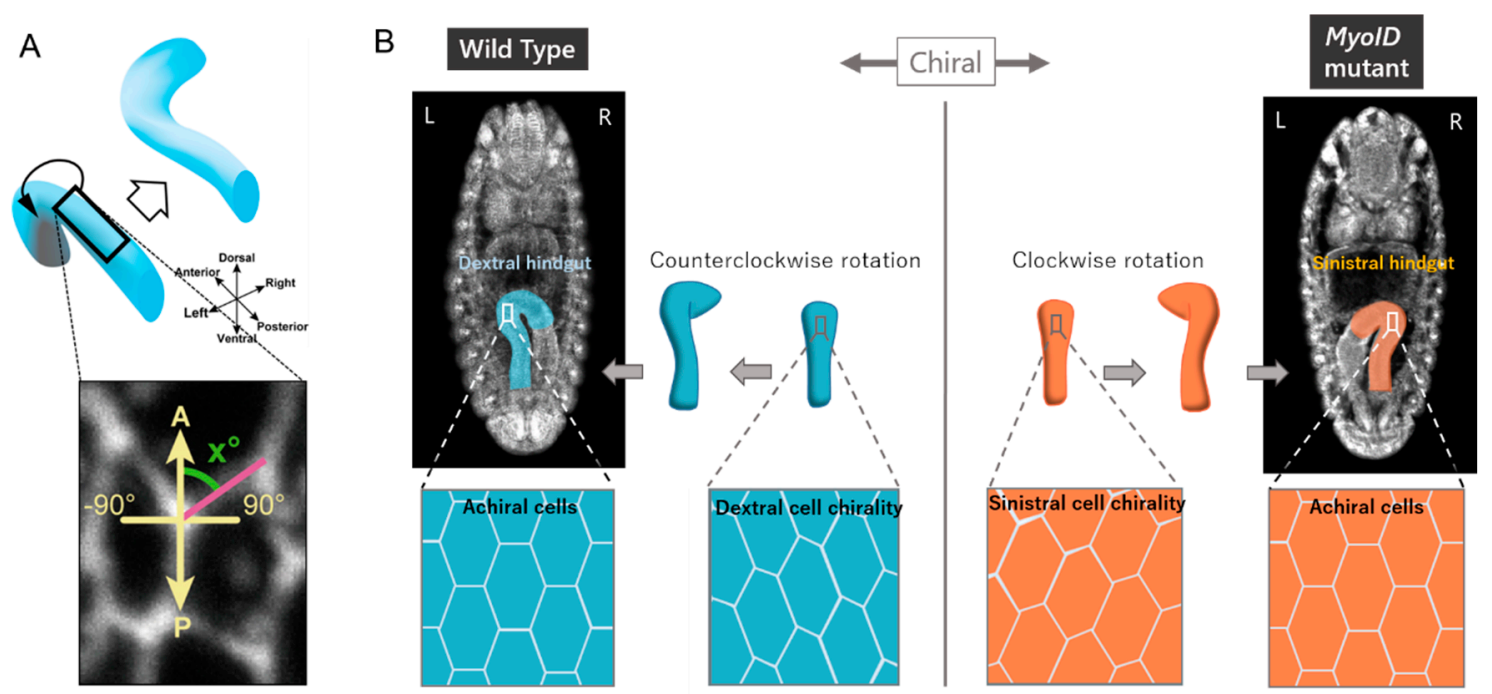

Figure 1. Enantiomorphic states (dextral and sinistral) of the cell chirality found in wild-type (left panel) and the MyoID mutant (right panel) are correlated with the direction of hindgut rotation observed in the Drosophila embryo [11]. (A) To detect cell chirality in the wild-type hindgut epithelium (shown by a black rectangle) before hindgut rotation (upper), the apical cell boundaries of the hindgut epithelial cells were visualized (bottom). The angle $X$ between the apical cell boundaries and the anterior-posterior axis of the hindgut (shown by A-P) was measured, and the left-right (LR) bias of the angle $X$ distribution was analyzed [11]. The anterior, posterior, dorsal, ventral, left, and right directions in the embryo are indicated. (B) Apical cell boundaries tend to slant to the left with respect to the anterior-posterior axis of the hindgut before hindgut rotation in wild type (left panel). In the MyoID mutant, in which the hindgut rotates sinistrally, the slanting of the cell boundaries becomes the mirror image of the wild type (right panel).

\section{Cellular Basis for LR Asymmetric Morphogenesis in Drosophila}

Studies on the mechanisms underlying LR asymmetric development in various invertebrates, including Drosophila, Caenorhabditis elegans, and Pulmonata snails, revealed the contributions of chirality at the cellular level $[11,29,30]$. Using Drosophila as a model, our group discovered cell chirality in vivo and revealed a connection between cell chirality and LR asymmetric development (Figure 1) [11]. This species has various organs that demonstrate directional LR asymmetry, including the embryonic and adult gut, testes, genitalia, and brain [31-35]. Among these organs, the embryonic gut is the first to develop LR asymmetry during embryogenesis [31]. The embryonic gut is composed of three major parts—the foregut, midgut, and hindgut -all of which show LR asymmetry in their structure and relative position in the body cavity [31]. LR defects in these structures are found in fewer than $1 \%$ of wild-type embryos, indicating that LR asymmetry is stringently determined [31].

The Drosophila embryonic hindgut has a simple tubular structure, which is suitable for studying the morphological changes occurring in its tissue and cells during LR asymmetric development. The embryonic hindgut is formed LR symmetrically at early stage 12, although its anterior part curves to the ventral side of the embryo and connects with the midgut (Figure 1) [11]. Subsequently, the embryonic hindgut rotates 90 degrees counterclockwise as viewed from the posterior end of the embryo, at stage 12-13, which causes the organ to curve to the right (Figure 1) [11]. To determine which tissue provides the active force that drives hindgut rotation, the hindgut was dissected out before its rotation, and cultured [14]. The cultured hindgut rotated just like in vivo, indicating that the active force driving its rotation comes from the hindgut itself, and not from surrounding organs or tissues in the embryo. The hindgut is composed of an epithelial tube and visceral muscles overlying it. The hindgut of a mutant in which the visceral muscles fail to differentiate and overlie the epithelial tube still rotates normally, indicating that the epithelial tube is sufficient to drive its own rotation [31]. In addition, cell proliferation does not occur in the hindgut during the rotation, nor does apoptosis 
contribute to this process [36,37]. Thus, the hindgut rotation was predicted to be induced by cell-shape changes and/or by rearrangements of hindgut epithelial cells, with LR asymmetry.

\section{Cell Chirality Drives LR Asymmetric Development in Drosophila}

The hindgut epithelium in Drosophila is a simple columnar epithelium that shows typical apical-basal polarity [31]. In the hindgut epithelium, the apical surface of each cell faces the lumen of the gut tube. In epithelia, the apical cell boundaries are often responsible for inducing epithelial morphogenesis $[38,39]$. To investigate this process, LR asymmetry in the shape of the apical cell boundaries was analyzed. Although the most anterior part of the hindgut curves ventrally before it rotates, the rest of this organ has a straight tubular structure [11]. Thus, the anterior-posterior axis of the tube can be determined accurately by analyzing micrograph images. Using these pictures, we measured the angle between the anterior-posterior axis and each cell boundary. The results in wild-type embryos showed that the apical cell boundaries slanted leftwards on average (Figure 1, left panel). The microscope images revealed that the apical surface of each hindgut epithelial cell was an ellipse, with its major axis running in the general direction of the anterior-posterior axis of the hindgut. These major axes slanted leftwards with respect to the anterior-posterior axis of this organ before its rotation [14]. This slanting was observed over the entire circumference of the hindgut tube. After the 90-degree counterclockwise rotation of the hindgut, the leftward slant of the cell boundaries and of the major axis of the apical-surface cells disappeared $[11,14]$. Considering that each hindgut epithelial cell has apical-basal polarity, these observations also indicate that the hindgut epithelial cells are chiral because their original image cannot be superimposed on their mirror image [11]. This chirality at the cellular level is called "planar cell chirality" [11]. After rotation of the hindgut, the cell chirality disappeared, and the hindgut epithelial cells became achiral [11,14].

The contribution of cell chirality to the embryonic hindgut rotation was demonstrated by computer simulations using two- and three-dimensional vertex models that recapitulated the apical cell boundaries of the hindgut epithelial tube $[11,14]$. In these vertex models, a contraction force that was LR asymmetrical with respect to the anterior-posterior axis of the model epithelium was introduced to recapitulate the cell chirality that is observed in vivo [11,14]. By setting certain conditions, the observed cell chirality could be replicated, at least to some extent, in this model epithelium [11,14]. Next, because the cell chirality disappeared after embryonic hindgut rotation in vivo [11], we introduced such a transition from chiral to achiral states as introduced into the computer simulation, and this transition induced the LR directional rotation of the model gut tube $[11,14]$. These results suggested that the loss of cell chirality could induce the LR-directional rotation of the hindgut [11,14]. We speculate that the dissolution of cell chirality induces a chiral change in the shape of the cells in the epithelial tube, which consequently drives tissue deformation leading to the counterclockwise rotation of the hindgut.

Drosophila melanogaster is a useful model organism in which genetic analyses can be performed to investigate various biological processes, including LR asymmetric development. Using this organism, we conducted a large-scale genetic screen to identify mutants affecting the LR asymmetric development of the embryonic gut. This screen identified MyosinID (MyoID) and Drosophila E-cadherin (DE-cad) as mutants that reversed and randomized the LR asymmetry of the embryonic hindgut, respectively (for the MyoID mutant, see Figure 1, right panel) [31]. MyoID is a conserved myosin I family protein (Figure 2) that is encoded by the Myosin31DF gene in Drosophila [40]. Strikingly, in homozygotes of various MyoID loss-of-function mutants, including a null allele, the LR asymmetry of the embryonic and adult hindgut, testes, and male genitalia were mirror images of their wild-type counterparts [31,32]. A null mutant of MyoID was homozygous viable and fertile, suggesting that MyoID has a specific role in LR asymmetric development, although it also contributes to other cellular events [41,42]. We analyzed the cell chirality of the hindgut epithelium in a MyoID homozygote and found that it also was the mirror image (sinistral) of its wild-type counterpart (dextral) (Figures 1 and 2) [11]. 


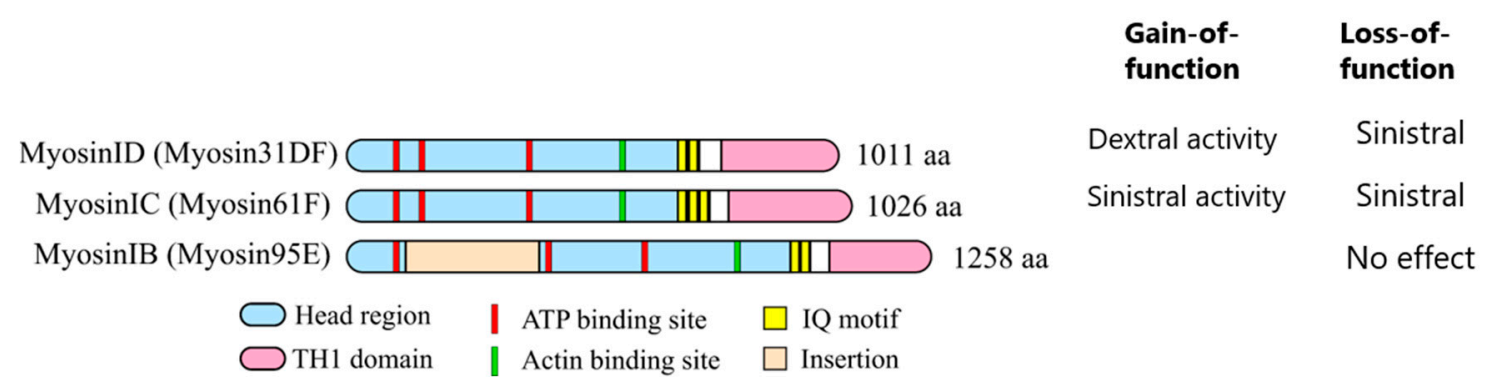

Figure 2. Structures of three myosin I proteins and their functions under gain- and loss-of-function conditions with respect to the LR asymmetry formation in Drosophila.

In addition, cell chirality was abolished in the hindgut epithelium of $D E$-cad homozygotes on average [11]. In the DE-cad mutants, the LR asymmetry of the hindgut is randomized [11]. We speculate that each embryo of this mutant still weakly shows either dextral or sinistral cell chirality, which can probably still drive hindgut rotation in either the left or right direction, depending on the enantiomorphic state of the cell chirality in the embryo. However, the cell chirality disappeared on average if many embryos were analyzed to represent the cell chirality of the $D E$-cad mutant. These results collectively suggest that the enantiomorphic state of the cell chirality determines the LR direction of the hindgut rotation (Figure 1) [11].

To determine whether this cell chirality forms cell intrinsically or not, we used genetic mosaicism. Overexpressing wild-type MyoID in the hindgut epithelium of a MyoID mutant completely rescued the defects in cell chirality formation and LR asymmetric development of the embryonic hindgut [11]. We then overexpressed wild-type MyoID in a subset of cells (rescued cells) in the hindgut epithelium of a MyoID homozygote, to generate a genetic mosaic hindgut epithelium composed of rescued cells and MyoID mutant cells. In this genetic mosaic hindgut, the rescued cells showed dextral cell chirality, while the surrounding MyoID mutant cells showed weak sinistral cell chirality [43]. These results indicate that cell chirality was formed cell intrinsically in each cell and was not induced by the tissue-level LR polarity [43]. This idea is consistent with recent findings that vertebrate cells in culture can exhibit cell chirality at the single-cell level [24,25].

The pivotal role of cell chirality in LR asymmetric development appears to be a general phenomenon in Drosophila [11-13]. As mentioned above, in the MyoID mutant, the LR asymmetry of various organs, including the embryonic and adult gut and the male genitalia, are the mirror images of those in wild type [31,32]. Kuranaga's group showed that the cell chirality of epithelial cells in the male genital disc is essential to drive the observed clockwise 360-degree rotation of the male genitalia [13]. Noselli's group reported that the cell chirality of adult gut cells is critical for the LR asymmetric looping of this organ [12]. Taken together, these findings indicate that cell chirality plays a general role in the LR asymmetric development in Drosophila.

\section{Cell Chirality Induces LR-Directional Cell Sliding in the Hindgut Epithelium}

We recently performed detailed analyses of live images depicting the positions of cell nuclei in the Drosophila embryonic hindgut epithelium during hindgut rotation and revealed how cell chirality induces rotation of this organ. These analyses revealed that the positions of cell nuclei with respect to those of their posterior neighbors slid in the direction of the hindgut rotation (Figure 3) [14]. In the wild-type and MyoID mutant hindguts, the nuclei slid in opposite directions, which corresponded to the counterclockwise and clockwise rotation of the organ, respectively [14]. This sliding of nuclei was observed throughout the entire circumference of the hindgut tube and indicated that the direction of nuclear sliding showed chirality and was correlated with the direction of the hindgut epithelial tube (Figure 3) [14]. Such changes in the relative position of nuclei could be induced by anisotropic cell-boundary remodeling, which is often involved in the deformation of epithelial tissue [38]. Our live imaging analyses also revealed that junctional remodeling occurred in these epithelial cells at a low 
frequency; approximately $10 \%$ of the cell junctions showed remodeling during the entire rotation of the hindgut [14]. However, the junctional remodeling did not show a detectable LR bias [14]. The observed junctional remodeling could probably be accounted for by the simultaneous convergent extension that is known to be required for axial elongation of the hindgut tube [44]. Based on these findings, we proposed that the sliding of nuclei reflects a change in the relative position of epithelial cells caused by changes in the chiral cell shape, without the need for LR asymmetric junctional remodeling [14]. We designated these novel cell dynamics as "cell sliding" [14].

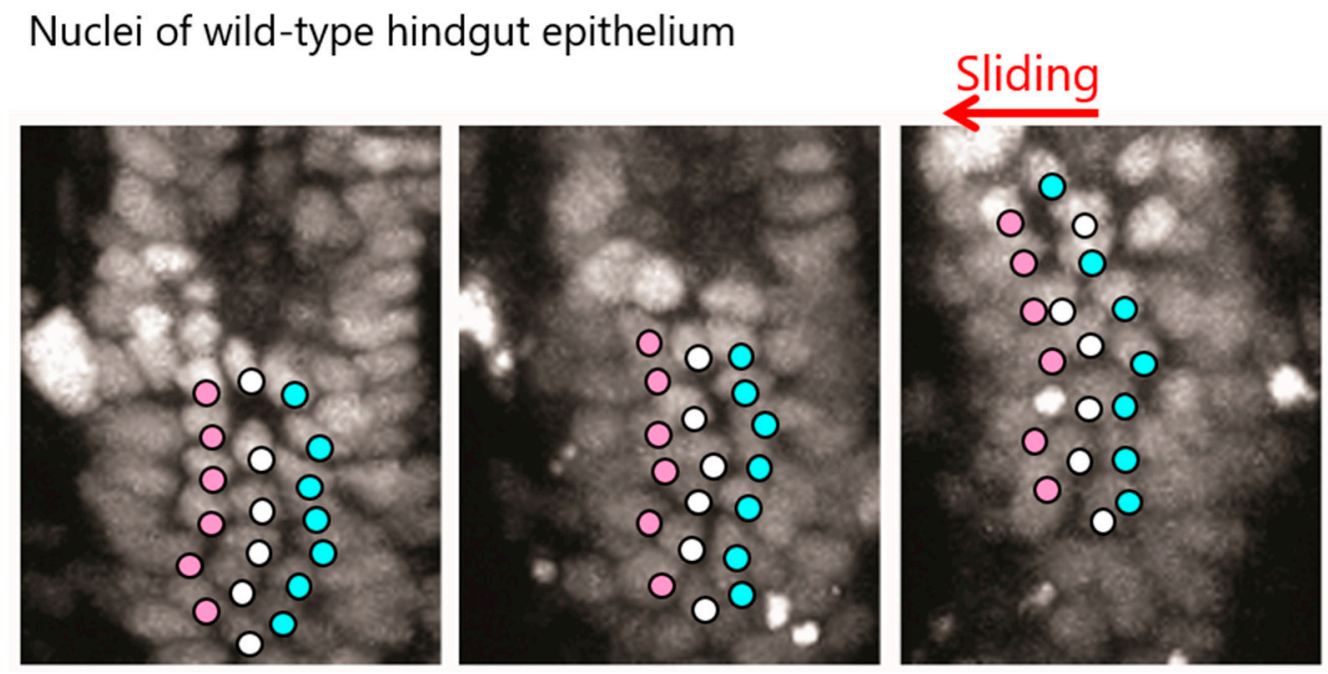

Cell slides though slow deformation of cell shape in simulation

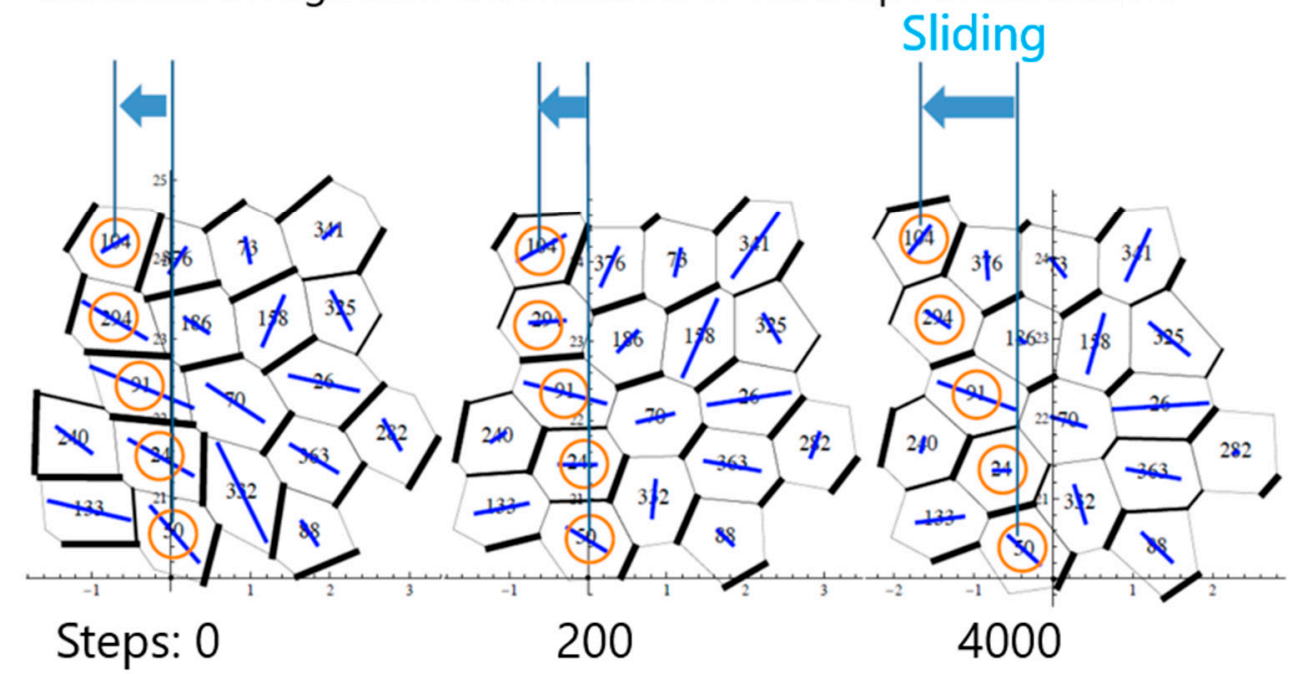

Figure 3. The dissolution of cell chirality induces cell sliding in vivo (upper) and in silico (lower) through slow deformations in the cell shapes.

These observations led us to hypothesize that the rotation of the hindgut epithelium is induced by the dissolution of cell chirality that was formed before hindgut rotation [11]. To test this possibility, we analyzed the relationship between cell chirality and cell sliding using a 3D vertex model, as described above. In this model, an LR-biased contraction force was applied to introduce chirality into the epithelial cells, and then neutralization of the LR-biased contraction force consequently induced LR directional rotation of the model gut tube [14]. After neutralization, the cell-shape change from chiral to achiral occurred rapidly (after only 200 steps), followed by slow cell sliding and tube rotation (4000 steps) (Figure 3) [14]. Thus, the timing of the initiation of gut tube rotation coincided with 
that of cell sliding but not with the rapid cancellation of the chiral cell shape, suggesting that cell sliding is the primary cause of gut tube rotation (Figure 3) [14]. In other words, these observations suggested that the dissolution of cell chirality is a mechanically rapid process, whereas cell sliding is a tissue deformation process that is much slower (Figure 3) [14]. On the other hand, the contribution of cell-boundary rearrangement is minor, although it was included in our vertex model. In a modified vertex model in which cell-boundary rearrangement was prohibited, but was otherwise the same as the original model, the cell sliding and gut tube rotation were induced similarly as in the original model [14]. Thus, cell sliding is not necessarily coupled with cell-boundary remodeling, although these events are not mutually exclusive. For example, in the male genitalia, cell chirality induces anisotropic cell-boundary remodeling that drives the rotation of an epithelial ring during the rotation of this organ [13]. In this system, the distribution of myosin II at the apical cell boundaries shows chirality, which introduces a chiral contraction force to the boundaries, consequently inducing anisotropic cell-boundary remodeling [13]. In contrast, cell-shape changes induce cell sliding in the hindgut epithelium, probably because this tissue is small enough to achieve its rotation without cell-boundary remodeling. Thus, cell sliding appears to be an intermediate cell behavior linking the dissolution of cell chirality with gut-tube rotation [14]. The dissolution of cell chirality induces a rapid alteration in cell shape, which is not directly linked to the hindgut rotation but is slowly converted into a chiral change in the relative positions of cells, which can be observed as a chiral tissue deformation, consequently resulting in hindgut rotation [14].

\section{Mechanisms of Cell Chirality Formation}

The mechanisms of cell chirality formation in Drosophila are beginning to be understood [10,11,43]. We previously proposed that a default state of LR asymmetry exists in this species, which was revealed in the MyoID mutant as the inversed LR asymmetry of various organs, including the embryonic and adult gut and the male genitalia [31,32]. In all cases investigated, inversed LR asymmetry has been coupled with a sinistral cell chirality in the organ $[11,13,45]$. Thus, the enantiomorphic state of the cell chirality is switched by MyoID, although MyoID is not required for the initial formation of sinistral cell chirality (Figure 2). These observations show that the sinistral state of cell chirality is the default condition in Drosophila [11]. A similar default chirality state was found in the blastomeres of Lymnaea stagnalis, a Pulmonata snail that maternally carries a formin gene mutation [8]. In this case, formin plays a similar role as MyoID in Drosophila. In Drosophila, the DE-cad gene was identified as another LR-asymmetry-related gene, as discussed above [11]. Notably, the distribution of the DE-cad protein at the adherens junctions of the hindgut epithelium shows dextral and sinistral chirality in wild-type and MyoID mutants, respectively [11]. We also revealed that DE-cad is epistatic to MyoID in the LR asymmetry phenotype of the embryonic hindgut, suggesting that the $D E$-cad protein functions downstream of the MyoID protein [11]. These results indicate that the formation of the default cell chirality requires $D E$-cad, although the molecular function of $D E$-cad in LR asymmetric development remains unclear [11].

Three myosin I-family genes, MyoID, Myosin IC (MyoIC), and MyosinIB (MyoIB), are present in the Drosophila genome (Figure 2) [45,46]. MyoID, MyoIC, and MyoIB are encoded by the Myo31DF, Myosin61F (Myo61F), and Myosin95E (Myo95E) genes, respectively (Figure 2). These three myosin proteins have conserved domains and motifs that are found in other myosin families, including a head region, ATP-binding site, TH1 domain, and IQ motif (Figure 2). Myo61F or Myo95E homozygotes without maternal contribution are viable and fertile and do not show any detectable phenotypes [45]. We generated triple homozygotes of the null mutants of all three of these genes lacking their maternal contributions and found that they too were viable and fertile [45]. This triple mutant did not show any detectable phenotypes except for the LR defects that were attributed to the MyoID mutation [45]. However, Myo61F enhanced the LR defect of the male genitalia rotation, suggesting that it has a redundant role with MyoID in the LR asymmetric development of this organ (Figure 2) [45]. Interestingly, the forced expression of Myo61F has a strong sinistral activity, which reverses the 
LR asymmetry of the embryonic gut and male genitalia (Figure 2) [31,47]. Thus, under their forced expression, MyoID and Myo61F have opposing activities in the LR asymmetric development of these organs [31,47]. We also observed that the sinistral activity of Myo61F is independent of MyoID, given that the LR inversion phenotype of the embryonic hindgut in a MyoID null mutant ( $80 \%$ LR inversion) was further enhanced by the forced expression of Myo61F in the hindgut epithelium [45]. However, these results are inconsistent with our finding that the Myo61F mutation enhances the LR defects associated with the MyoID mutant, as discussed above (Figure 2) [45]. Thus, the sinistral activity of Myo61F induced by its forced expression may not be physiological, although it was useful for dissecting the function of the domains in MyoID and Myo61F. Experiments in which the equivalent domains of the MyoID and Myo61F proteins were swapped revealed that the head domains but not the tail ones are responsible for the dextral and sinistral activities of MyoID and Myo61F, respectively $[10,48]$. The importance of the head region suggests that an interaction between F-actin and these myosin I proteins has an essential role in switching the enantiomorphic states of the cell chirality. A link between F-actin and myosin I was shown in an elegant biochemical experiment in which the motility of F-actin induced by membrane-bound Myo1c, a mammalian homolog of Myo61F, was observed [49]. This experiment revealed that the F-actin motility occurs along a curved path in a counterclockwise direction [49]. However, while Drosophila MyoID (Myo1D ortholog) induces this curved motility of F-actin, Drosophila Myo61F does not [10]. Based on these observations and the finding that the forced expression of MyoID but not Myo61F in certain tissues induces cell and organ chirality [10], it was proposed that the curved motility of F-actin driven by MyoID is the origin of the chirality that eventually leads to cell and body chirality. In summary, the chiral behavior of F-actin with respect to the plasma membrane may be the source of cell chirality or a molecular switch for the enantiomorphic state of cell chirality.

In addition to myosin I family proteins, various other regulators of F-actin have been shown to contribute to the formation of cell chirality [8,25]. For example, in mammalian cultured cells, the level of $\alpha$-actinin-1 switches the enantiomorphic state of the cell chirality [25]. The $\alpha$-actinin-1 protein binds to F-actin and induces F-actin bundle formation [50]. In zebrafish, melanophores exhibit cellular chirality, which is recognized as a unidirectional counterclockwise rotational movement under isolated conditions [24]. This counterclockwise rotation is suppressed by inhibiting the actin cytoskeleton but is enhanced by inhibiting microtubules [24]. Finally, as mentioned above, in Lymnaea stagnalis, the formin gene is mutated in individuals demonstrating disrupted cell chirality in four-cell stage blastomeres, which leads to LR inversion of the whole body [8]. Formin family proteins bind to the barbed end of actin filaments and nucleate actin polymerization [51]. During actin polymerization, formin undergoes a directional rotation at the barbed end [52]. These observations together suggest that the molecular chirality of F-actin plays a leading role in the formation of cell chirality. This is also likely to be the case in the hindgut epithelial cells of Drosophila, although the mechanisms by which such molecular chirality is integrated at the single-cell level remain unclear. Furthermore, the dissolution of cell chirality may also be controlled by the regulation of F-action function. At some points in these processes, MyoIC and MyoID can reverse the enantiomorphic state of the cell chirality.

\section{Perspectives}

In the LR asymmetric development of vertebrates, the LR axis is established in the embryo through the LR asymmetric distribution of extracellular signaling molecules at an early stage, when the embryo is still LR symmetric [53-55]. On the other hand, in Drosophila, such LR symmetry in the distribution of signaling molecules has not been reported. Furthermore, at the early stage when the embryo is LR symmetric, LR asymmetric gene expression has not been reported. Thus, we speculate that the LR axis may not form in the Drosophila embryo. On the other hand, cells in various tissues that eventually demonstrate LR asymmetry have intrinsic cell chirality in this species. These cells, which have apical-basal polarity, are located at various positions with respect to the anterior-posterior and dorsal-ventral axes, and their cell chirality can dictate directional LR asymmetric morphogenesis 
in individual organs in an organ-intrinsic manner. At this point, it is not clear how common this tissue-intrinsic mechanism of LR asymmetric morphogenesis is in animal development.

Although cell chirality is broadly observed in cultured vertebrate cells, its functions have just begun to be revealed in vitro if not in vivo in these species. Cell chirality has roles in vertebrate LR asymmetric development in parallel with other known mechanisms, such as nodal flow and LR asymmetric proton influx [56,57]. For example, chick cardiac cells show an intrinsic cell chirality that is responsible for the LR asymmetry break in cardiac looping [17]. In addition, cell chirality may be involved in the concordant organization of tissue, because the coexistence of cells with different enantiomorphic states of cell chirality within a tissue may introduce disharmony into the tissue's organization. It was also found that the chirality of mammalian endothelial cells regulates the integrity of their intercellular junctions, which affects endothelial permeability [58]. The identification of defects caused by abnormal states of cell chirality in vertebrates is a major upcoming challenge in this compelling field.

Author Contributions: S.U., S.S., T.S., T.I., C.M., M.I., and K.M. wrote the manuscript. S.U., S.S., C.M., and M.I. made the figures.

Funding: This work was supported by JSPS KAKENHI Grants \#JP15H05863 to K.M. \#JP16K07349 to T.S., and \#JP15K07077 to M.I.

Conflicts of Interest: The authors declare no conflict of interest.

\section{References}

1. Genchi, G. An overview on D-amino acids. Amino Acids 2017, 49, 1521-1533. [CrossRef] [PubMed]

2. Blackmond, D.G. The origin of biological homochirality. Cold Spring Harb Perspect. Biol. 2010, 2, a002147. [CrossRef] [PubMed]

3. Watson, T. An account of some cases of transposition observed in the human body. Lond. Med. Gaz. 1836, 18, 393-403.

4. Spemann, H.; Falkenberg, H. Uber Asymmetrische Entwicklung und Situs inversus viscerum bei Zwillingen und Doppelbildungen. Wilhelm Roux' Arch. Entwicklungsmech. Org. 1919, 45, 371-422. [CrossRef]

5. Okumura, T.; Utsuno, H.; Kuroda, J.; Gittenberger, E.; Asami, T.; Matsuno, K. The development and evolution of left-right asymmetry in invertebrates: Lessons from Drosophila and snails. Dev. Dyn. 2008, 237, 3497-3515. [CrossRef]

6. Sturtevant, A.H. Inheritance of direction of coilling in limnaea. Science 1923, 58, 269-270. [CrossRef]

7. Nonaka, S.; Yoshiba, S.; Watanabe, D.; Ikeuchi, S.; Goto, T.; Marshall, W.F.; Hamada, H. De novo formation of left-right asymmetry by posterior tilt of nodal cilia. PLoS Biol. 2005, 3, e268. [CrossRef] [PubMed]

8. Davison, A.; McDowell, G.S.; Holden, J.M.; Johnson, H.F.; Koutsovoulos, G.D.; Liu, M.M.; Hulpiau, P.; Van Roy, F.; Wade, C.M.; Banerjee, R.; et al. Formin Is Associated with Left-Right Asymmetry in the Pond Snail and the Frog. Curr. Biol. 2016, 26, 654-660. [CrossRef] [PubMed]

9. Kuroda, R.; Fujikura, K.; Abe, M.; Hosoiri, Y.; Asakawa, S.; Shimizu, M.; Umeda, S.; Ichikawa, F.; Takahashi, H. Diaphanous gene mutation affects spiral cleavage and chirality in snails. Sci. Rep. 2016, 6, 34809. [CrossRef]

10. Lebreton, G.; Géminard, C.; Lapraz, F.; Pyrpassopoulos, S.; Cerezo, D.; Spéder, P.; Ostap, E.M.; Noselli, S. Molecular to organismal chirality is induced by the conserved myosin 1D. Science 2018, 362, 949-952. [CrossRef]

11. Taniguchi, K.; Maeda, R.; Ando, T.; Okumura, T.; Nakazawa, N.; Hatori, R.; Nakamura, M.; Hozumi, S.; Fujiwara, H.; Matsuno, K. Chirality in planar cell shape contributes to left-right asymmetric epithelial morphogenesis. Science 2011, 333, 339-341. [CrossRef]

12. González-Morales, N.; Géminard, C.; Lebreton, G.; Cerezo, D.; Coutelis, J.-B.; Noselli, S. The Atypical Cadherin Dachsous Controls Left-Right Asymmetry in Drosophila. Dev. Cell 2015, 33, 675-689. [CrossRef]

13. Sato, K.; Hiraiwa, T.; Maekawa, E.; Isomura, A.; Shibata, T.; Kuranaga, E. Left-right asymmetric cell intercalation drives directional collective cell movement in epithelial morphogenesis. Nat. Commun. 2015, 6, 10074. [CrossRef]

14. Inaki, M.; Hatori, R.; Nakazawa, N.; Okumura, T.; Ishibashi, T.; Kikuta, J.; Ishii, M.; Matsuno, K.; Honda, H. Chiral cell sliding drives left-right asymmetric organ twisting. eLife 2018, 7. [CrossRef] 
15. Inaki, M.; Liu, J.; Matsuno, K. Cell chirality: Its origin and roles in left-right asymmetric development. Philos. Trans. R. Soc. Lond B Biol. Sci. 2016, 371. [CrossRef]

16. Inaki, M.; Sasamura, T.; Matsuno, K. Cell Chirality Drives Left-Right Asymmetric Morphogenesis. Front Cell Dev. Biol. 2018, 6, 34. [CrossRef]

17. Ray, P.; Chin, A.S.; Worley, K.E.; Fan, J.; Kaur, G.; Wu, M.; Wan, L.Q. Intrinsic cellular chirality regulates left-right symmetry breaking during cardiac looping. Proc. Natl. Acad. Sci. USA 2018. [CrossRef] [PubMed]

18. Gail, M.H.; Boone, C.W. The locomotion of mouse fibroblasts in tissue culture. Biophys. J. 1970, 10, 980-993. [CrossRef]

19. Hagmann, J. Pattern formation and handedness in the cytoskeleton of human platelets. Proc. Natl. Acad. Sci. USA 1993, 90, 3280-3283. [CrossRef]

20. Xu, J.; Van Keymeulen, A.; Wakida, N.M.; Carlton, P.; Berns, M.W.; Bourne, H.R. Polarity reveals intrinsic cell chirality. Proc. Natl. Acad. Sci. USA 2007, 104, 9296-9300. [CrossRef] [PubMed]

21. Tamada, A.; Kawase, S.; Murakami, F.; Kamiguchi, H. Autonomous right-screw rotation of growth cone filopodia drives neurite turning. J. Cell Biol. 2010, 188, 429-441. [CrossRef]

22. Wan, L.Q.; Ronaldson, K.; Park, M.; Taylor, G.; Zhang, Y.; Gimble, J.M.; Vunjak-Novakovic, G. Micropatterned mammalian cells exhibit phenotype-specific left-right asymmetry. Proc. Natl. Acad. Sci. USA 2011, 108, 12295-12300. [CrossRef]

23. Chen, T.-H.; Hsu, J.J.; Zhao, X.; Guo, C.; Wong, M.N.; Huang, Y.; Li, Z.; Garfinkel, A.; Ho, C.-M.; Tintut, Y.; et al. Left-Right Symmetry Breaking in Tissue Morphogenesis via Cytoskeletal MechanicsNovelty and Significance. Circ. Res. 2012, 110, 551-559. [CrossRef] [PubMed]

24. Yamanaka, H.; Kondo, S. Rotating pigment cells exhibit an intrinsic chirality. Genes Cells 2015, 20, 29-35. [CrossRef]

25. Tee, Y.H.; Shemesh, T.; Thiagarajan, V.; Hariadi, R.F.; Anderson, K.L.; Page, C.; Volkmann, N.; Hanein, D.; Sivaramakrishnan, S.; Kozlov, M.M.; et al. Cellular chirality arising from the self-organization of the actin cytoskeleton. Nat. Cell Biol. 2015, 17, 445-457. [CrossRef]

26. Tamada, A.; Igarashi, M. Revealing chiral cell motility by 3D Riesz transform-differential interference contrast microscopy and computational kinematic analysis. Nat. Commun. 2017, 8, 2194. [CrossRef]

27. Wan, L.Q.; Chin, A.S.; Worley, K.E.; Ray, P. Cell chirality: Emergence of asymmetry from cell culture. Philos. Trans. R. Soc. Lond B Biol. Sci. 2016, 371. [CrossRef]

28. Satir, P. Chirality of the cytoskeleton in the origins of cellular asymmetry. Philos. Trans. R. Soc. Lond B Biol. Sci. 2016, 371. [CrossRef]

29. Kuroda, R.; Endo, B.; Abe, M.; Shimizu, M. Chiral blastomere arrangement dictates zygotic left-right asymmetry pathway in snails. Nature 2009, 462, 790-794. [CrossRef]

30. Naganathan, S.R.; Fürthauer, S.; Nishikawa, M.; Jülicher, F.; Grill, S.W. Active torque generation by the actomyosin cell cortex drives left-right symmetry breaking. eLife 2014, 3, e04165. [CrossRef]

31. Hozumi, S.; Maeda, R.; Taniguchi, K.; Kanai, M.; Shirakabe, S.; Sasamura, T.; Spéder, P.; Noselli, S.; Aigaki, T.; Murakami, R.; et al. An unconventional myosin in Drosophila reverses the default handedness in visceral organs. Nature 2006, 440, 798-802. [CrossRef]

32. Speder, P.; Adam, G.; Noselli, S. Type ID unconventional myosin controls left-right asymmetry in Drosophila. Nature 2006, 440, 803-807. [CrossRef] [PubMed]

33. Kozopas, K.M.; Samos, C.H.; Nusse, R. DWnt-2, a Drosophila Wnt gene required for the development of the male reproductive tract, specifies a sexually dimorphic cell fate. Genes Dev. 1998, 12, 1155-1165. [CrossRef] [PubMed]

34. Adám, G.; Perrimon, N.; Noselli, S. The retinoic-like juvenile hormone controls the looping of left-right asymmetric organs in Drosophila. Development 2003, 130, 2397-2406. [CrossRef]

35. Pascual, A.; Huang, K.L.; Neveu, J.; Préat, T. Neuroanatomy: Brain asymmetry and long-term memory. Nature 2004, 427, 605-606. [CrossRef] [PubMed]

36. Lengyel, J.A.; Iwaki, D.D. It takes guts: The Drosophila hindgut as a model system for organogenesis. Dev. Biol. 2002, 243, 1-19. [CrossRef]

37. Wells, R.E.; Barry, J.D.; Warrington, S.J.; Cuhlmann, S.; Evans, P.; Huber, W.; Strutt, D.; Zeidler, M.P. Control of tissue morphology by Fasciclin III-mediated intercellular adhesion. Development 2013, 140, 3858-3868. [CrossRef] [PubMed] 
38. Bertet, C.; Sulak, L.; Lecuit, T. Myosin-dependent junction remodelling controls planar cell intercalation and axis elongation. Nature 2004, 429, 667-671. [CrossRef] [PubMed]

39. Blankenship, J.T.; Backovic, S.T.; Sanny, J.S.; Weitz, O.; Zallen, J.A. Multicellular rosette formation links planar cell polarity to tissue morphogenesis. Dev. Cell 2006, 11, 459-470. [CrossRef]

40. Morgan, N.S.; Skovronsky, D.M.; Artavanis-Tsakonas, S.; Mooseker, M.S. The molecular cloning and characterization of Drosophila melanogaster myosin-IA and myosin-IB. J. Mol. Biol. 1994, 239, 347-356. [CrossRef]

41. Amcheslavsky, A.; Wang, S.; Fogarty, C.E.; Lindblad, J.L.; Fan, Y.; Bergmann, A. Plasma Membrane Localization of Apoptotic Caspases for Non-apoptotic Functions. Dev. Cell 2018, 45, 450-464.e3. [CrossRef]

42. Packard, M.; Jokhi, V.; Ding, B.; Ruiz-Canada, C.; Ashley, J.; Budnik, V. Nucleus to Synapse Nesprin1 Railroad Tracks Direct Synapse Maturation through RNA Localization. Neuron 2015, 86, 1015-1028. [CrossRef]

43. Hatori, R.; Ando, T.; Sasamura, T.; Nakazawa, N.; Nakamura, M.; Taniguchi, K.; Hozumi, S.; Kikuta, J.; Ishii, M.; Matsuno, K. Left-right asymmetry is formed in individual cells by intrinsic cell chirality. Mech. Dev. 2014, 133, 146-162. [CrossRef]

44. Iwaki, D.D.; Lengyel, J.A. A Delta-Notch signaling border regulated by Engrailed/Invected repression specifies boundary cells in the Drosophila hindgut. Mech. Dev. 2002, 114, 71-84. [CrossRef]

45. Okumura, T.; Sasamura, T.; Inatomi, M.; Hozumi, S.; Nakamura, M.; Hatori, R.; Taniguchi, K.; Nakazawa, N.; Suzuki, E.; Maeda, R.; et al. Class I Myosins Have Overlapping and Specialized Functions in Left-Right Asymmetric Development in Drosophila. Genetics 2015, 199, 1183-1199. [CrossRef]

46. Tzolovsky, G.; Millo, H.; Pathirana, S.; Wood, T.; Bownes, M. Identification and phylogenetic analysis of Drosophila melanogaster myosins. Mol. Biol. Evol. 2002, 19, 1041-1052. [CrossRef]

47. Petzoldt, A.G.; Coutelis, J.B.; Géminard, C.; Spéder, P.; Suzanne, M.; Cerezo, D.; Noselli, S. DE-Cadherin regulates unconventional Myosin ID and Myosin IC in Drosophila left-right asymmetry establishment. Development 2012, 139, 1874-1884. [CrossRef]

48. Hozumi, S.; Maeda, R.; Taniguchi-Kanai, M.; Okumura, T.; Taniguchi, K.; Kawakatsu, Y.; Nakazawa, N.; Hatori, R.; Matsuno, K. Head region of unconventional myosin I family members is responsible for the organ-specificity of their roles in left-right polarity in Drosophila. Dev. Dyn. 2008, 237, 3528-3537. [CrossRef]

49. Pyrpassopoulos, S.; Feeser, E.A.; Mazerik, J.N.; Tyska, M.J.; Ostap, E.M. Membrane-bound myo1c powers asymmetric motility of actin filaments. Curr. Biol. 2012, 22, 1688-1692. [CrossRef]

50. Tojkander, S.; Gateva, G.; Lappalainen, P. Actin stress fibers-Assembly, dynamics and biological roles. J. Cell Sci. 2012, 125 Pt 8, 1855-1864. [CrossRef]

51. Higgs, H.N. Formin proteins: A domain-based approach. Trends Biochem. Sci. 2005, 30, 342-353. [CrossRef]

52. Mizuno, H.; Higashida, C.; Yuan, Y.; Ishizaki, T.; Narumiya, S.; Watanabe, N. Rotational movement of the formin mDia1 along the double helical strand of an actin filament. Science 2011, 331, 80-83. [CrossRef]

53. Levin, M.; Johnson, R.L.; Stern, C.D.; Kuehn, M.; Tabin, C. A molecular pathway determining left-right asymmetry in chick embryogenesis. Cell 1995, 82, 803-814. [CrossRef]

54. Collignon, J.; Varlet, I.; Robertson, E.J. Relationship between asymmetric nodal expression and the direction of embryonic turning. Nature 1996, 381, 155-158. [CrossRef]

55. Lowe, L.A.; Supp, D.M.; Sampath, K.; Yokoyama, T.; Wright, C.V.; Potter, S.S.; Overbeek, P.; Kuehn, M.R. Conserved left-right asymmetry of nodal expression and alterations in murine situs inversus. Nature 1996, 381, 158-161. [CrossRef]

56. Nonaka, S.; Tanaka, Y.; Okada, Y.; Takeda, S.; Harada, A.; Kanai, Y.; Kido, M.; Hirokawa, N. Randomization of left-right asymmetry due to loss of nodal cilia generating leftward flow of extraembryonic fluid in mice lacking KIF3B motor protein. Cell 1998, 95, 829-837. [CrossRef]

57. Levin, M.; Thorlin, T.; Robinson, K.R.; Nogi, T.; Mercola, M. Asymmetries in H+/K+-ATPase and cell membrane potentials comprise a very early step in left-right patterning. Cell 2002, 111, 77-89. [CrossRef]

58. Fan, J.; Ray, P.; Lu, Y.; Kaur, G.; Schwarz, J.J.; Wan, L.Q. Cell chirality regulates intercellular junctions and endothelial permeability. Sci. Adv. 2018, 4, eaat2111. [CrossRef]

(C) 2019 by the authors. Licensee MDPI, Basel, Switzerland. This article is an open access article distributed under the terms and conditions of the Creative Commons Attribution (CC BY) license (http:/ / creativecommons.org/licenses/by/4.0/). 